Session 2793

\title{
A Literature Review on the Under-representation of Women in Undergraduate Engineering: Ability, Self-Efficacy, and the "Chilly Climate"
}

\author{
David Malicky \\ University of Kansas
}

\section{Introduction}

The low retention of undergraduate women in engineering (WIE), combined with their low application rates, results in a continuing under-representation of women in the industrial and academic engineering culture. From 1987 to 1997, the undergraduate enrollment of women in engineering increased from $15 \%$ to $19 \%$, in a relatively consistent but gradual manner ${ }^{1}$. In science and engineering combined, women now earn $47 \%$ of bachelor degrees, though they are overrepresented in some fields such as psychology and underrepresented in others such as computer science and most fields of engineering. Among minorities, women earn more than half of science and engineering degrees. Farrell ${ }^{2}$ notes that the proportion of women engineers of the past 20 years has only grown $5 \%$, despite a number of mentorship programs, scholarships, and curricular improvements throughout the country. One engineering dean describes it as "a national crisis" ( $p$. A31).

The under-representation of women in science, mathematics, and engineering (SME) fields is important for many reasons. The future productivity of the US workforce depends on attracting greater numbers of scientists and engineers: the current workforce is aging at the same time that job skills are becoming increasingly technical ${ }^{3}$. The shortfall of women in SME fields has at least two implications for this productivity. First, they represent an untapped reservoir of potential employees, and second, they may bring new perspectives and ideas to meeting new challenges ${ }^{4-6}$.

Beyond the economic and productivity implications are social and ethical motivations. Scientific literacy is increasingly important to health and environmental issues. But the understanding of SME fundamentals by most Americans - particularly women due to their under-representation in $\mathrm{SME}$ - is inadequate to fully participate in these issues ${ }^{7}$. According to the National Research Council $^{7}$, "Our nation is becoming divided into a technologically knowledgeable elite and a disadvantaged majority" (p. 1), with women representing a disproportionate part of that majority. In 1972, Federal Title IX legislation prohibited sex discrimination in education, yet there is evidence of subtle discriminatory practices in SME culture ${ }^{8-12}$.

Representation in undergraduate studies is a function of two mechanisms: enrollment and persistence. Many interventions have advocated strong recruitment efforts to improve women's enrollment in SME, but these have often resulted in disappointing cost/benefit outcomes: enrollments have grown, but only by about $1 \%$ per year, and retention of underrepresented groups continues to lag behind the majority ${ }^{13}$. The reasons are not far to seek: "if programs addressing under-representation are primarily shaped by a search for undiscovered talent, while the structural 
and cultural barriers to enrollment and persistence among under-represented groups remain obscure or unaddressed, such programs cannot succeed" (Seymour ${ }^{13}$, p. 8). And there is an ethical dimension to the choice to focus on recruitment: while controversial, many studies show that the university SME environment is unfriendly, even hostile, to women engineers. Thus, is it ethical to recruit women into a field of study that they are likely to find discriminatory? Yet, representation will continue to be a problem if no recruitment efforts are performed. A resolution can be found in the manner of "recruitment" employed. If "recruitment" is done with a "push-in" approach — marketing SME to women and girls despite its differentially inhospitable environment - then those women may unduly suffer and the "pipeline" will develop "leaks." But if "recruitment" is thought of as a "pull-in"—changing the undergraduate SME environment so that it treats women fairly and is even attractive to them - then representation is increased without undue risk of suffering and "leaks."

An engineering analogy applies: to increase the flow in a pipe, one could 1) apply more pressure to the supply side of the pipe or 2) increase the diameter of the pipe. The former results in excess stress on the medium, strain on the pipe, and leaks. The latter just results in more flow, though it does require a new pipe. The required changes to increase the women-friendliness of SME are considerably more complex than increasing the diameter of a pipe. Thus, the first step in improving representation is to understand the dimensions of women's experiences in the SME culture: social, cultural, academic, interpersonal, and intrapersonal.

There are a multitude of factors, forces, and structures associated with women's underrepresentation in undergraduate SME - more than can be covered in one literature review. This review examines three factors which are among the best understood and considered most important: ability, self-efficacy, and discrimination. To put these in context, a full literature review would also include these topics: expectations, enjoyment, contextualized knowledge, congruence, role-conflict, competition, group work, and support. The focus of this review is on the undergraduate years, though it provides a brief overview of pre-college factors to inform the undergraduate experience. This focus is not to downplay the importance of the pre-college, graduate school, faculty, or industry research literatures on WIE; these must be understood for an overall assessment of women's experiences in engineering.

This review aims to stay as close to the actual research data as possible. Author's interpretations are given less weight than actual results. Whether null hypotheses are rejected or not rejected, those results are reported and incorporated with the other results for a particular topic. When factors are found to be significant, an attempt is made to quantify that factor in some way, either by the level of the difference or its correlation to another factor.

This review focuses specifically on engineering where possible, though it draws upon the larger and more thorough SME literature as it is relevant in most respects. The literature on women in engineering originates from many disciplines: sociology, education, psychology, women's studies, linguistics, career counseling, human development, government, and engineering. This review aims to integrate the empirical findings from these diverse viewpoints, especially as they apply to retention. The primary articles of this review were selected based on relevance, empirical rigor, and variety of scientific methods. 


\section{Gender Differences in Persistence}

There is little question that matriculation decisions are primarily responsible for the underrepresentation of women in engineering. But what role does persistence play? Meaningful retention data are difficult to obtain for a variety of reasons such as accounting for transfer students, declaration variances, and tracking duration. In a recent study designed to overcome these difficulties, Adelman ${ }^{14}$ analyzed transcripts of 8,395 students ( $9 \%$ of which were engineers) over 11 years. In engineering, retention of men was nearly $20 \%$ higher than that of women: $61.6 \%$ v. $41.9 \%$. Disproportionate attrition combines with disproportionate matriculation to result in women accounting for as little as $10.4 \%$ of degree completers in engineering. This $20 \%$ difference is considerably higher than previous work based on CIRP (Cooperative Institutional Research Program $\left.{ }^{15}\right)$ data ${ }^{13}$ or that in highly-selective institutions ${ }^{16}$.

\section{Pre-College Experiences Of Males And Females}

The origins of women's under-representation in SME can be traced to the pre-college socialization of boys and girls ${ }^{17}$. Studies have shown the science and math experiences, confidence, and ability of boys and girls are generally quite similar. Leslie ${ }^{17}$ provides a review of this literature. But, in adolescence, males and females diverge in their confidence and their experiences are characterized by different expectations, attention, and support, usually in favor of males (for a review of this literature, see Seymour ${ }^{8}$ and Leslie ${ }^{17}$ ). Seymour ${ }^{8}$ finds that in high school, strong differences emerge between men and women in their reasons for choosing their major: women were more influenced by family and high school teachers, and had less clear ideas about the major itself and its career path. Pre-college girls often received high praise from their science teachers, but sometimes were stigmatized for being good in science, which led to lower aspirations and doubts about belonging in this field.

Leslie $^{17}$ identifies the critical period influencing women's later choices in SME as adolescence, with some seeds planted in childhood. General self-concept forms at an early age though early socializing forces are relatively similar for boys and girls. During adolescence, self-concepts differentiate into specific math and science self-efficacies. Family members and adolescent peers are key influences on this differentiation. As a result, females graduate high school with fewer math-science courses and lower aspirations and commitments to SME careers.

\section{Study Methodologies}

The literature on undergraduate WIE spans many methodologies, settings, participants, and aims. Most of the studies of this review are quantitative, some qualitative, a few use both methodologies, and one is a meta-analysis. Most compare women to men, others focus on women. (Unless stated otherwise in this paper, "women" refers to women in engineering, and "men" to men in engineering.) Argument-based articles are introduced in the rest of this review as appropriate, though the focus remains on empirical findings. Examining the methodologies of the central sources of this review, there are:

- 7 qualitative and 21 quantitative studies (including mixed methods for both)

- 13 single institution and 12 multi-institution studies

- 13 cross-sectional, 11 longitudinal, and 1 meta-analysis

- 20 mixed gender and 5 female-only studies

- Approximately 37,000 undergraduate participants, $39 \%$ of which are female. 
These data indicate a well balanced variety of methods and settings, good representation in both genders, and a very large number of participants. With this level of examination of a topic, findings of consistency should be worthy of considerable confidence.

\section{Academic Ability: Qualifications, Spatial Skills, And Achievement}

For the purposes of this review, academic qualifications refers to students' credentials on entry into college: e.g., HS GPA, math/science level, SAT scores. Academic achievement refers to incollege factors: GPA, credits earned, and test scores. Academic ability is often used for any prior qualifications or achievement in predicting a future outcome. This section addresses these basic questions:

1. Are there gender differences in qualifications?

2. Are there gender differences in visualization skills?

3. Are there gender differences in achievement?

4. If so, (how) do these differences affect retention?

1. Qualifications: Overall, women have equal or slightly better qualifications than their male peers.

The most comprehensive study of pre-college qualification is found in Adelman ${ }^{14}$, who analyzed transcripts and other credentials of a national database of high school students entering college. He found that the high school math/science backgrounds of $1^{\text {st }}$ year engineers were very similar for men and women. For more women than men, their highest math level was calculus, precalculus, or trigonometry, but these advantages were not significant (2-8\% differences). Correspondingly, more men's than women's highest level was algebra 2, which was significant (12\% difference). In science, there were no significant gender differences in the number of years of HS coursework. SAT scores of women entering engineering exceeded the men's by a small margin (1093 v. 1016).

These findings are similar to those of Felder ${ }^{18}$, who found no significant gender differences in SAT scores or AP credits for North Carolina State University chemical engineering students. Studying 197 engineering students at one university, Hackett ${ }^{19}$ found women were advantaged with higher HS GPAs (3.8 v. 3.6), but there were no significant differences in SAT scores. Using self-ratings of preparation in math, chemistry, physics, and biology, Haines' study ${ }^{10}$ at the University of Calgary only found significant differences for biology, with the advantage to women. At the U. of Colorado, women entering engineering had slightly higher predicted-GPA than men $(3.05 \mathrm{v}$. 2.99 $)^{20}$.

Overall, women's qualifications appear to be equal or slightly better than men's.

2. Spatial skills: Overall, men are advantaged in spatial skills and previous experience, but these differences can be eliminated by training.

Spatial skills have been examined as a predictor of engineering performance. Three types of spatial ability have been identified: spatial perception, mental rotation, and spatial visualization ${ }^{21}$. 
$\mathrm{Hyde}^{21}$ reviewed meta-analyses on gender differences (for the entire population, not only engineers), computing effect sizes (standard deviation / difference in means) to quantify any differences, when present. Overall, men were advantaged in spatial ability with a mean effect size, $\mathrm{d}$, of 0.45 (range: $\mathrm{d}=0.13$ to 0.73 , the largest being mental rotation).

Devon $^{22}$ also found initial gender differences in mental rotation, but these were substantially reduced by training: the percentage of variance explained by gender dropped from $15 \%$ before training to $5 \%$ after training. Perhaps for the same reason, that study found mental rotation scores were not related to retention.

Sorby ${ }^{23}$ examined spatial skills for students at 3 universities in the US and Europe. Gender differences were found at all settings, significantly favoring males, ranging from $12-29 \%$. In the US university only, males had more previous experience in drafting.

Overall, there appear to be gender differences in spatial ability and previous experience, but additional training reduces these differences to negligible levels.

\section{Achievement: Women appear to perform substantially similar to men.}

Adelman ${ }^{14}$ found women carry a small but significantly higher credit load than do men $(30.7 \mathrm{v}$. 29.8 hours per year). Grades of women and men are generally similar, with women performing better in mechanical engineering, industrial engineering, and engineering math, and men performing better in computer engineering (significance was not assessed for overall GPA). Grandy $^{24}$ studied SME students nationally taking the GRE (i.e., seeking graduate school). In all major field groups, men scored higher than women in the GRE general test, by a 0.7 effect size. GPA differences for this select group favored men, but by a small margin. Takahira ${ }^{25}$ examined course grades in Statics across 17 diverse institutions; no gender difference was found.

Three single institution studies corroborate these findings. Felder ${ }^{18}$ found that at the end of the $1^{\text {st }}$ year, grades were not gendered except in English (favoring women). In their sophomore and junior chemical engineering courses, the men received equal or higher grades than the women, with significance obtained in 2 of 5 courses. But, overall GPA after both the sophomore and senior years was not gendered. Hughes ${ }^{11}$ also found no gender differences in the GPA of sciencemath-technology majors. Hackett ${ }^{19}$ developed a regression model using gender and rigorously operationalized social cognitive factors to predict achievement (GPA, not retention). Their model yielded close prediction of cumulative GPA $(R=0.73)$. Yet, gender was not one of the significant predictors in this model.

Overall, there are no notable gender trends for undergraduate academic achievement except that women and men perform substantially the same.

\section{Is retention predicted by gender? Mixed results.}

A number of studies have attempted to predict retention using ability, gender, and other factors. With the inclusion of other factors, results are mixed with respect to the influence of gender on 
the prediction of retention in engineering.

No: Using the CIRP database, $\operatorname{Sax}^{26}$ developed a blocked stepwise regression model of 330 potential factors to predict retention in SME. These national data show retention is predicted by ability and preparation, but these factors did not significantly differ for men and women.

In predicting retention at four highly selective universities, Strenta ${ }^{27}$ found overall retention was gendered in engineering. $(56.8 \% \mathrm{~F}$ v. $64.7 \% \mathrm{M})$ but that gender was not a significant predictor when ability and performance variables were controlled. This implies women's ability and performance measures were lower than men's - a trend not generally seen in the studies above or at Berkeley ${ }^{20}$. Gender was a significant predictor for math and the physical sciences in Strenta's study.

In a single-institution study, Schaefers ${ }^{4}$ developed a hierarchical logistic regression model using ability and carefully operationalized psychological variables to predict retention. Overall, $92.6 \%$ of persisters and $62.3 \%$ of nonpersisters were correctly predicted-67\% better than chance. Persistence rates were not significantly different for women (69\%) and men (73\%). The most significant predictor variable was ability, with the student's first semester GPA nearly as effective as all ability variables combined. Neither gender nor any 2-way interactions with gender were significant predictors.

Yes: Adelman's analysis ${ }^{14}$ of national data did find gender differences between persisters and nonpersisters: Compared to male non persisters, female nonpersisters had higher college grades and lower SAT test scores than their male peers, though their high school backgrounds were not significantly different.

Takahira $^{25}$ examined persistence within a single course (Statics) and across 17 diverse institutions and found some gender trends. Overall, men were slightly more likely to persist (effect size $=$ 0.08). Persistence in Statics was correlated to GPA (especially for women) and SAT-math (especially for men). Compared to men, women were less likely to persist in Statics as institutional selectivity increases. This last finding is consistent with Strenta's results for the entire major, above, but is contrary to results at Berkeley ${ }^{16}$ where gender differences were nearly eliminated at that highly selective institution.

Gender was also important for Univ. of Colorado engineering students. Both women persisters and nonpersisters had higher predicted-GPA than the corresponding $\operatorname{men}^{20}$.

At the Univ. of Washington, Brainard ${ }^{28}$ examined differences in women's GPAs for persisters and nonpersisters. At the time of switching, no GPA differences were found, indicating that women are not leaving because they are not performing - a finding reflected in Seymour and Hewitt.

In sum, there are national database studies showing both gender effects (Adelman) and no gender effects (Sax) on retention. And, there are multi-institutional studies on both sides (Takahira v. Strenta). And, there are single institution studies on both sides (McClelland v. Schaefers). This controversy can be better understood after examining self-efficacy and a reconciliation is 
attempted at the end of this review as part of a theoretical model.

\section{Self-Efficacy: Gender, Correlates, Persistence, \& Engineering Culture}

Self-efficacy ${ }^{29}$ is situation-specific self-confidence; it is the belief in one's competence and skills to achieve in a given activity, such as mathematics, engineering, public speaking, or athletics. Selfefficacy is a belief in one's capacity to perform a particular task, whereas self-confidence tends to be a more general concept of self-worth. Nevertheless, they are often correlated and in many studies, not distinguished. In his original study, Bandura showed that self-efficacy accurately predicted performance in $85 \%$ of the tasks confronted. Later studies have continued to validate the role of self-efficacy in predicting behavior ${ }^{30}$. Self-efficacy is thought of as a mediator between ability and actual performance ${ }^{31}$. That is, one may have high ability, but without self-efficacy one's performance will suffer. Conversely, self-efficacy is also thought to be based on past performance, as well as vicarious experience, verbal persuasion, and emotional activation ${ }^{29}$.

Self-efficacy has been heavily studied for WIE. This section addresses four questions:

1. Are there gender differences in SME self-efficacy?

2. What are the dynamics of SME self-efficacy for women?

3. What are the correlating factors (predictors and effects) for SME self-efficacy?

4. Is persistence predicted by SME self-efficacy?

1. Gender differences in SME self-efficacy: Women appear to have lower SME self-efficacy than their male peers, by a moderate amount.

A number of studies have compared the self-efficacy of men and women in SME fields ${ }^{4,10,11,17-19 \text {, }}$ $24,27,32,33$. Of these, three ${ }^{4,31,33}$ did not find significant gender differences in SME self-efficacy; the other seven did find such differences, favoring men. These 10 studies are described below, starting with the multi-institutional studies.

Besterfield-Sacre ${ }^{32}$ employed a validated instrument to survey 13 attitudes of $1^{\text {st }}$ year engineering students at 17 institutions. Of these 13 attitudes, 5 were measures of self-efficacy. These measures and the associated results over the first year are:

1. Basic engineering knowledge and skills: Women $<$ Men, significant at $82 \%$ of schools pre$1^{\text {st }}$ year and $47 \%$ of schools post- $1^{\text {st }}$ year.

2. Communication and computer skills: No trend.

3. Study habits (arguably not SME self-efficacy but "effort"): Mixed results. Women $>$ Men pre- $1^{\text {st }}$ year, significant at $35 \%$ of schools. Post- $1^{\text {st }}$ year there is no trend.

4. Problem solving abilities: Women $<$ Men, significant at $71 \%$ of schools pre- $1^{\text {st }}$ year and $47 \%$ of schools post- $1^{\text {st }}$ year.

5. Engineering abilities: Women $<$ Men, significant at $100 \%$ of schools pre- $1^{\text {st }}$ year and $87 \%$ of schools post- $1^{\text {st }}$ year. On a 1-5 scale, the average amount of difference is approximately 0.3 - the largest and most consistent gender difference in any of the 13 attitudes over the first year.

The trend from the above findings is that men are generally advantaged for the 3 self-efficacy measures most relevant to undergraduate $\operatorname{SME}(1,4$, and 5). 
Grandy $^{24}$ examined 13 self-evaluated skill measures for SME seniors taking the GRE. For SME students overall, men's self-efficacy was higher than women's for 3 of the 13 skills: problem solving, mathematics, and test-taking. For the entire SME sample, women's self-efficacy was higher than men's for 2 skills: organizing work and time spent on homework (again, arguably "effort"). For the other 8 self-efficacy measures, women and men rated themselves quite similarly. Among this sample of SME students, the largest differences were seen for engineering students, with moderate to large effect sizes favoring men for laboratory skills, test taking abilities, and ability to think through problems.

Leslie $^{17}$ analyzed students in the CIRP database (not only SME) and found gender and racial differences for SME self-efficacy. At entry to college, students who self-rated their SME preparation as "better than most" (p. 256) had race x gender characteristics as shown in Table 1. Whether White, Black, or Hispanic, women consistently rate their SME preparation lower than their male peers. Leslie found the self-efficacy advantage of white males carried through for students in SME fields, though specific data were not reported.

Table 1: Percentage of students in CIRP database rating their SME self-efficacy as "better than most," by race and gender, after Leslie ${ }^{17}$.

\begin{tabular}{|c|c|c|c|}
\hline & Men (all CIRP) & Women (all CIRP) & Difference \\
\hline White & $43 \%$ & $31 \%$ & $12 \%$ \\
\hline Black & $26 \%$ & $16 \%$ & $10 \%$ \\
\hline Hispanic & $23 \%$ & $17 \%$ & $6 \%$ \\
\hline
\end{tabular}

Self-efficacy differences extended to students at highly selective institutions. Strenta ${ }^{27}$ surveyed SME majors for three self-efficacy measures among other data. For engineering students, men had significantly better self-efficacy for all three measures (a 0-3 rating scale was used for each; note direction of "better" is variable):

1. Question ability to handle coursework: Men $<$ Women by 0.27 points.

2. Feel confident speaking in class: Men $>$ Women by 0.48 points.

3. Feel depressed about academic progress: Men $<$ Women by 0.29 points. Strenta then correlated feelings of confidence and depression to science and non-science GPA. He found science GPA, but not non-science GPA, was a "highly significant negative predictor" (p. 531) of confidence and depression for engineering students. Strenta assumed that causality was from GPA to self-efficacy, though it is likely the reverse could be true as well.

Somewhat mixed results are seen in five single institution studies. At a commuter university in Canada, Haines ${ }^{10}$ surveyed 5 student attitudes (preparation, performance, interest, ease, and confidence) related to choice of an engineering major across four HS subjects (math, chemistry, physics, and biology). All the attitudes except interest represented self-efficacy. All subjects except biology are central for all engineering students. The remaining 4 attitudes and 3 subjects give 12 highly relevant self-efficacy measures. Only 2 of these showed effect sizes greater than 0.2 : physics ease $(\mathrm{d}=0.33$ favoring men $)$ and physics confidence $(\mathrm{d}=0.39$ favoring men $)$. Women did have higher self-efficacy in biology preparation $(\mathrm{d}=0.29)$ and biology performance $(\mathrm{d}=0.25)$, notably consistent with their high representation in bioengineering. Haines also examined certain self-efficacy measures affecting persistence and progress in engineering: mathematics, chemistry, 
physics, engineering, and spatial tasks. Of these 5 measures, women rated their abilities lower than their male peers for 3 : physics $(d=0.29)$, engineering $(d=0.23)$, and spatial tasks $(d=0.30)$.

At Georgia Southern university, Hughes ${ }^{11}$ examined course confidence for science, mathematics, and technology majors. While not specifically surveying engineers, this study is important because it uniquely examines course confidence as a function of the gender of both student and instructor. With instructors of either gender, women had significantly less course confidence than men despite similar GPAs (confidence differences of $0.2-0.5$ on a 5 point scale). For female students only, course confidence was

1. Negatively correlated to a male instructor showing favoritism to either male or female students $(\mathrm{p}<0.05$, correlation coefficients -0.16 to -0.21$)$.

2. Positively correlated to respect from male peers, in a class with a female instructor $(\mathrm{p}<0.05$, correlation coefficient 0.28$)$. This finding supports research on discrimination, below.

Felder ${ }^{18}$ tracked men and women chemical engineers throughout the engineering curriculum. In their sophomore year, females rated their academic preparation lower than their male peers, with $24 \%$ of women and $45 \%$ of men rating their preparation as "strong." In their junior and senior years, women continued to rate their abilities to solve basic engineering problems lower than men, with $20 \%$ differentials common. Less consistent were differences in creative problem solving selfefficacy, which were significant only at the end of the senior year. All of these disadvantages for self-efficacy existed despite women having equal or better GPAs, as seen in Hughes, above.

Hackett's ${ }^{19}$ regression model of achievement also provided gender comparisons for two rigorously operationalized measures: SME occupational self-efficacy and academic milestones self-efficacy (academic milestones refers to completing 12 core requirements of the engineering program). Reliability coefficients of $0.8-0.9$ are typical for the instruments employed in this study and validity is based on established psychological theories. For the 197 students sampled, neither measure showed significant gender differences (for comparison to Grandy ${ }^{24}$ and Haines ${ }^{10}$, effect sizes favoring men of approximately 0.3 can be calculated from Hackett's data, for both measures).

Using a rigorous self-efficacy operationalization similar to Hackett, Schaefers ${ }^{4}$ also found no gender effects.

The academic self-efficacy measures in Meinholdt's ${ }^{33}$ study were based on Hackett's. A trend favoring men was found for the 150 students sampled $(p<0.07)$. No gender differences were found for vocational self-efficacy.

What can we conclude about gender differences and self-efficacy, based on the above studies? If we give the most weight to the cross-institutional studies, self-efficacy differences not only exist, they are usually among the largest gender differences for attitudes and beliefs. However, if we give the most weight to studies using validated measures, the picture is somewhat less clear. Besterfield-Sacre ${ }^{34}$ is the only such study that supports a gender difference; Hackett ${ }^{19}$, Schaefers ${ }^{4}$, and Meinholdt ${ }^{33}$ are three such studies (all single institution) that do not. And, if we give the most 
weight to studies using reliable and valid measures based closely on established psychological theory ${ }^{4,19,33}$, gender differences in SME self-efficacy are highly questionable. These disparities raise important questions for future research, such as whether the latter three studies are operationalizing self-efficacy significantly differently than other studies, and whether the other studies are measuring something other than SME self-efficacy. This difficulty also underscores the importance of assessing and documenting an instrument's reliability and validity. Considering:

1. The high inherent face-validity of typical self-confidence/self-efficacy measures.

2. Meinholdt ${ }^{33}$ found a trend $(p=.07)$ for academic (but not vocational) self-efficacy favoring men.

3. Hackett's data ${ }^{19}$ indicates an effect size favoring men comparable to multi-institutional studies (though based on that sample it was not significant).

4. In no case was women's SME self-efficacy higher than men's.

It seems prudent to at least tentatively conclude that self-efficacy gender differences exist, they favor men, and they are of small to moderate effect sizes.

2. Dynamics of SME self-efficacy and self-esteem: Women begin college with high selfefficacy, lose a marked portion early in their $1^{\text {st }}$ year, and regain some of that loss in their senior year.

For both men and women, self-efficacy is believed to be one of the key factors in a student's achievement and persistence. Additional research has focused not necessarily on gender differences, but on qualitatively and quantitatively characterizing the changes in self-efficacy over time, and the forces on and effects of self-efficacy. Unfortunately, some research does not carefully distinguish between self-efficacy, overall self-confidence, and self-esteem. As these three attributes are likely to be correlated, all applicable data are reported and the results are phrased consistent with the original study.

Brainard ${ }^{28}$ longitudinally examined the experiences of women at the University of Washington. Women began college with high self-confidence in their academic abilities: on a 1-5 scale, mean self confidence for math and science were 4.01 and 3.98, respectively. These levels dropped significantly over the $1^{\text {st }}$ year to 3.37 and 3.52 , respectively. Self-efficacy remained near this level until the end of their senior year, when they rebounded to the 3.7 range. The drop in academic selfefficacy in the $1^{\text {st }}$ year is corroborated by 3 studies: Arnold ${ }^{35}$, Seymour \& Hewitt ${ }^{13}$, and Anderson ${ }^{5}$, outlined below.

Arnold $^{35}$ found the self-esteem and self-confidence of women to drop over the freshman year. But, for men over this same period, self-esteem rose and self-confidence was unchanged. Besterfield-Sacre's data would seem to be in conflict with Arnold's findings: the former study found gender differences to be reduced over the 1st year.

The landmark ethnographic study of Seymour and Hewitt ${ }^{13}$ (gender specific findings also in Seymour $^{8}$ ) provide strong support for the drop in women's self-confidence over the 1st year (recall that all students in this study had SAT scores of 650 or above):

... most women we encountered had entered college at peak of self-confidence, based on good high

Proceedings of the 2003 American Society for Engineering Education Annual Conference \& Exposition Copyright (C) 2003, American Society for Engineering Education 
school performances, good or adequate SAT scores, and a great deal of encouragement and praise from high school teachers, family, and friends. Within a relatively short time of their entry to college, women who felt intelligent, confident in their abilities and prior performance level, and who took their sense of identity for granted, began to feel isolated, insecure, intimidated, to question whether they "belonged" in the sciences and all, and whether they were good enough to continue (Seymour, 1995, p.458).

Anderson's ${ }^{5}$ grounded theory study also confirms the loss in self-confidence and self-esteem from the beginning of the $1^{\text {st }}$ year, though this applied only to some of the interviewees. Most women experienced only "temporary discouragement and self-doubt" (p. 103), and a few women moved through their 4 years without "any apparent loss of self-esteem" (p. 103).

The data in Felder ${ }^{18}$ provides some confirmation of Brainard's findings ${ }^{28}$ that self-efficacy increases over the senior year. Women rating their basic problem solving abilities as "excellent" or "good" increased from their junior to the end of their senior year, from $68 \%$ to $75 \%$. Creative problem solving self-efficacy showed an even larger increase, from $25 \%$ to $44 \%$.

3. Correlating factors for SME self-efficacy: Women's SME self-efficacy is most consistently correlated to achievement (GPA) and to positive influences of faculty and role-models.

Many studies have correlated self-efficacy to performance, psychological, and external factors. For most of the correlating factors, it cannot be precisely determined whether the factor is a predictor, an effect, or both, though causation in one direction is often assumed.

Brainard ${ }^{28}$ correlated math and science self-confidence to a number of measures. The most consistent measures were 1) high rating of instruction math and science, 2) positive influence of math and science classes and instructors (as seen in Hughes, below), and 3) persistence. Further results from Brainard's study are discussed below.

For all students and all instructors in Hughes' study ${ }^{11}$, course confidence was positively correlated to recognition by and respect from the instructor $(\mathrm{p}<0.01$, correlation coefficients ranging from 0.23 to 0.52 ). Interestingly, women's course confidence was not significantly correlated to GPA, indicating that course self-efficacy is obtained on a course by course basis.

Nauta $^{36}$ developed a structural equation model incorporating ability, self-efficacy, role model influence, and role conflict to predict the career aspirations of women in SME. This model assumed ability and role-model-influence to predict self-efficacy, which then predicts higher level career aspirations. Conservatively, it would be best to think of "predict" as "correlate," since causality may occur both ways. Working with their hypothesized model, ability (operationalized as SAT and GPA, with GPA most predictive) predicted self-efficacy with a pathway coefficient of 0.54 for SME (non-biology) students. Positive role models similarly predicted self-efficacy with a path coefficient of 0.33 . All paths were significant at $p<0.05$.

In Hackett's model ${ }^{19}$, occupational and academic self-efficacy were significantly correlated to a wide range of variables. Overall self-efficacy is most related to GPA, engineering interests, expectations, stress/strain/coping, and faculty encouragement or discouragement. Two variables

Proceedings of the 2003 American Society for Engineering Education Annual Conference \& Exposition Copyright $(\mathrm{C}$ 2003, American Society for Engineering Education 
were not significant predictors: HS GPA and family/peer support. SAT scores were weak predictors. Hackett's population was upper level students; it would be interesting to examine whether the HS GPA and SAT factors would be predictors for $1^{\text {st }}$ year students. The insignificance of family/peer support is not well understood. The findings of Nauta and Brainard indicate consistency for the dimensions of GPA and the role of faculty as role models/ encouragement sources.

4. Is persistence predicted by self-efficacy? Low self-efficacy is an independent predictor of nonpersistence; low achievement compounds that risk.

Four studies have investigated the "bottom-line" question: does self-efficacy predict persistence? Unlike the correlating factors above where the arrow of causality may run both ways, such as for GPA, the end-state nature of persistence allows us to assume any causal connection found is from self-efficacy to persistence.

Three of the studies ${ }^{13,26,27}$ are multi-institution; the others are single institution. Recall in Brainard's single institution study ${ }^{28}$ persistence was consistently predicted by math-science selfconfidence, and that these self-confidence measures dropped markedly over the $1^{\text {st }}$ year and remained depressed until the senior year. But when students are surveyed for their perceptions of barriers to persistence, those citing "lack of self-confidence" increase dramatically in the senior year (from $23-27 \%$ in $1^{\text {st }}$-Junior years to $45 \%$ in the senior year). Thus, it appears that while average self-confidence is rebounding in the senior year, more seniors are concerned about their low level of self-confidence. Recall that in comparing persisters and nonpersisters, Brainard did not find GPA differences at the time of switching. But self-confidence differences were found: in science through all four years and also in math in the sophomore year $(\mathrm{p}<.05$ for all). Brainard also surveyed nonpersisters for their reasons for switching. Of the 6 reasons cited, two are related to self-efficacy: "conceptual difficulties" and "discouraged by low grades" (p. 374). Both factors could be functions of both ability and self-efficacy; but since nonpersisters had the same GPA as persisters, it is likely these do reflect self-efficacy. "Conceptual difficulties" was cited as a reason for switching in 37\%-45\% of cases; "Discouraged by low grades" in $41 \%-60 \%$. In the sophomore year - the peak of attrition problems - both factors peak in frequency and in rank with respect to the other 6 reasons (ranking $3^{\text {rd }}$ and $1^{\text {st }}$, respectively). Low math self-efficacy peaks this year as well. This evidence - that students most frequently cite low self-efficacy as a reason for switching at the same time that they are switching most frequently-suggests that self-efficacy was a primary reason for not persisting (this latter analysis of Brainard's data is not found in that report.)

Schaefer's ${ }^{4}$ model of upper level students utilized gender, ability, self-efficacy, and other variables to predict persistence. The strongest predictor was ability (operationalized as $1^{\text {st }}$ term GPA, cumulative GPA, and ACT-Math) ( $<<0.001)$, followed by math-science self-efficacy $(\mathrm{p}<0.001)$. Recall that gender was not a significant predictor in this study.

Sax's analysis ${ }^{26}$ of CIRP data show self-rating of math ability is a significant predictor (beta $=.02$ to .12) of retention and this factor did not significantly differ for men and women. Also, women (but not men) who take more math courses in college are more likely to persist (beta $=.12$ ), 
suggesting this may be related to the lower math confidence women experience.

Strenta $^{27}$ surveyed nonpersisters and found $41 \%$ of male and $48 \%$ of female students (not significantly different) cited "work in science was too difficult" as a reason for leaving - a measure of low self-efficacy. Strenta apparently did not attempt to incorporate the self-efficacy measures in his retention model.

Seymour and Hewitt ${ }^{13}$ included a quantitative component to their ethnographic study, though statistical comparisons were not made. Of most relevance to this review, they report factors contributing to switching decisions specifically for engineering students. Recall their population was screened with a minimum SAT-Math of 650. Reasons for switching that are related to low self-efficacy are shown in Table 2. Their results indicate that self-efficacy is important towards persistence (Nos. 8, 12, 13, and 15), though less so than suggested by Brainard and Schaefer. Instead, interest, workload, and teaching/advising variables appear to play larger roles.

Table 2: Seymour and Hewitt's compilation ${ }^{13}$ of reasons for switching from engineering. The first two columns are added for this review; the rest is directly from Seymour and Hewitt (p. 46).

\begin{tabular}{|c|l|l|c|c|c|}
\hline & Genre & Issue & $\begin{array}{c}\text { Factor in } \\
\text { Switching } \\
\text { Decisions } \\
(\%)\end{array}$ & $\begin{array}{c}\text { All } \\
\text { Switchers } \\
\text { concerns } \\
(\%)\end{array}$ & $\begin{array}{c}\text { All non- } \\
\text { switchers } \\
\text { concerns } \\
(\%)\end{array}$ \\
\hline 1 & Interest & $\begin{array}{l}\text { Lack of/loss of interest in SME: "turned off by } \\
\text { science" }\end{array}$ & 50 & 41 \\
\hline 2 & Workload & Curriculum overload, fast-paced overwhelming & 45 & 55 & 52 \\
\hline 3 & Teaching & Poor teaching by SME faculty & 41 & 98 & 86 \\
\hline 4 & Interest & $\begin{array}{l}\text { Non-SME major offers better education/more } \\
\text { interest }\end{array}$ & 37 & 57 & 35 \\
\hline 5 & Workload & $\begin{array}{l}\text { SME career options/rewards felt not worth effort } \\
\text { for degree }\end{array}$ & 31 & 43 & 18 \\
\hline 6 & Interest & Shift to more appealing non-SME career & 30 & 36 & 14 \\
\hline 7 & $\begin{array}{l}\text { Advising } \\
\text { Inadequate advising or help with academic } \\
\text { problems }\end{array}$ & $\begin{array}{l}\text { Discouraged/lost confidence due to low grades in } \\
\text { early years }\end{array}$ & 25 & 81 & 53 \\
\hline 8 & $\begin{array}{l}\text { Self- } \\
\text { efficacy }\end{array}$ & Rejection of SME careers and as sociated lifestyles & 24 & 44 & 29 \\
\hline 9 & Interest & $\begin{array}{l}\text { Reasons for choice of SME major prove } \\
\text { inappropriate }\end{array}$ & 20 & 94 & 52 \\
\hline 10 & Motive \\
\hline 11 & Financial & Financial problems of completing SME majors & 18 & 32 & 29 \\
\hline 12 & $\begin{array}{l}\text { Self- } \\
\text { efficacy }\end{array}$ & Morale undermined by competitive SME culture & 16 & 30 & 9 \\
\hline 13 & $\begin{array}{l}\text { Self- } \\
\text { efficacy }\end{array}$ & Conceptual difficulties with SME subject(s) & 15 & 32 & 29 \\
\hline 14 & Support & Lack of peer study group support & 14 & 19 & 12 \\
\hline 15 & $\begin{array}{l}\text { Self- } \\
\text { ability }\end{array}$ & Inadequate HS preparation in subjects/study skills & 10 & 38 & 37 \\
\hline
\end{tabular}

Proceedings of the 2003 American Society for Engineering Education Annual Conference \& Exposition Copyright $(\mathrm{C}$ 2003, American Society for Engineering Education 
Future research could examine the inter-relationships between interests and self-efficacy. Possibly, students may be more likely to report loss of interest than loss of self-efficacy (due to either unawareness and/or impression management), and self-efficacy losses may cause loss of interest.

From these quantitative studies, it appears that regardless of gender, students experiencing low self-efficacy have significantly higher risk of attrition. This risk exists even with grades equal to persisters, though lower grades increase the risk dramatically. Future research may examine the reasons for the discrepancies in the above data. Potential reasons may include

- Differences in types of self-efficacy

- Differences in methods of self-efficacy operationalization

- Low reliability for the chosen self-efficacy operationalization

- Excessively general/ambiguous self-efficacy measures

- Differences in student populations, by ability, year, and location

- Researcher bias.

\section{Self-Efficacy Of Women In An Engineering Culture}

Seymour and Hewitt's ${ }^{13}$ qualitative data seem to emphasize self-efficacy somewhat more so than their quantitative survey of the same students. But more importantly, their qualitative data provide insight into the complex mechanisms of self-efficacy dynamics - mechanisms which may be missed by quantitative research. This section explores two of the dynamics of self-efficacy for these women:

1. Interrelationship of past and current support with socialization

2. Interplay of performance demands, self-concept, and male student's expectations

\section{Interrelationship of support, performance expectations, and socialization}

Past support from high school teachers, in the form of accolades, interacts with college faculty expectations about performance, in the form of "trials," to downgrade women's self-efficacy. Many women experienced bolstering support from their high school science teachers and depended on them for reassurance and motivation. They began college at peak of self-esteem (confirmed by Brainard ${ }^{28}$ ). But, in college, this support was almost completely withdrawn and expectations were raised, leading to anxiety, frustration, and a severe drop in confidence. Many engineering faculty have instituted a "weed-out" system that challenges engineers to survive in a harsh climate. Seymour and Hewitt liken it to a "rite of passage." Most men students understand this challenge on some level due to their socialization of competitiveness. But women are socialized differently, and simply do not perceive the motives for this test of which they are being subjected. It is outside their conceptual framework to understand why they are being treated with both high demands and little support/feedback. Thus the pressures worked to heighten women's anxiety, whereas for men the pressures heightened their determination. Those women that learned to depend on themselves for evaluation and motivation tended to progress in SME. Those that did not tended to leave. Some, especially seniors, became consciously aware of the system:

The average of all our exams are in the '40s, so, if the men in the class get a $\mathrm{C}$, they're happy. If women get a $\mathrm{C}$, they're upset. They think the teacher doesn't think they're any good. And they lineup outside his door trying to get him to explain it, or tell them, that's [sic] it's really okay. I think that's a very difficult thing for women to get over at first. (Seymour ${ }^{8}$, p. 466, 
white engineering nonswitcher).

You just feel so overwhelmed that you have to prove yourself to the men... The proving process goes on and on. It takes years. I still feel like I'm battling the whole male ego. (Seymour ${ }^{8}$, p. 461, women science senior).

Expecting support and reassurance and receiving an obscurely motivated "weed-out" process, it is not surprising women would question their skills and place in engineering.

\section{Relationship of performance demands, male student's expectations, and self-concept.} The second self-efficacy dynamic is the interplay of those demands to perform, women students' self-concept, and male students' expectations about women. Women negotiate "conflicting" roles of being accomplished, being attractive, and fitting in:

I think a lot of guys carry this assumption that women in techy fields are just unattractive... It's some sort of conceptual shift. And it's not open for discussion. (Seymour ${ }^{8}$, p. 454, male science switcher)

I would like to strangle half the students at times. When I first came here, they wouldn't let me touch anything in the lab. They let me take down the data -- that's all. The last day of class, they let me weigh something. (Seymour ${ }^{8}$, p. 457, female engineering switcher)

We were at a party recently, and another engineer in her class asked my friend, "So, are you sleeping with the professor? Is that how you do it?" (Seymour ${ }^{8}$, p. 456)

For at least some male students in engineering, then, are these expectations of women:

- A woman cannot be both attractive and in engineering.

- Women are not competent in engineering.

Perhaps as a result, women were often targets of unkind and sexually overtoned remarks/jokes that made women feel uncomfortable and unwelcome (see Tonso ${ }^{37}$ for clear examples of this). Women were also targets of attack by some men when they performed well. From a focus group of women seniors:

I think you tend not to want to tell them that you did well on a test.

Right. When they give back the tests, if you get a good grade, you slip it into your folder, because, heaven forbid you should do well.

They're jealous if you do well -- 'cause we shouldn't be smarter than they are. (Seymour ${ }^{8}, \mathrm{p}$. 455)

Many women negotiated these expectations and attitudes by attempting to become neutral and invisible:

There's a perceived threat that feeds male anger about bright women. People tell jokes that are negative to women, which just fuels it. And it encourages the women to pull back into safer ground - to choose more traditional majors, or to adopt a more neutral style — as a way 
of protecting themselves from the anger. (Seymour ${ }^{8}$, p. 456, female science nonswitcher).

They try to play down their femininity -- not to show it as much -- so they won't get picked on. The attitude against them, it's so overt. (Seymour ${ }^{8}$, p. 454, male science nonswitcher)

Overall, then, at least some male engineering students are sending these messages to women students:

- "I am better than you. If you seem better, it's not for a legitimate reason."

- "You don't belong here. If you are here, you must be unattractive or should be invisible."

It is not surprising that self-efficacy would suffer in such a pride-depriving environment This review now focuses on further studies of discrimination and disrespect that characterize this environment.

\section{The "Chilly Climate" For Women In Engineering}

The hypothesis of a "chilly climate" for women in the classroom has been developed in the larger higher education literature ${ }^{38-40}$. Whitt's findings ${ }^{40}$ indicate that female perceptions of gender-based discrimination exist on college campuses: e.g., $43 \%$ of students disagree or strongly disagree with the statement, "I have never observed discriminatory words, behaviors, or gestures directed towards female students" (p.166). Whitt finds these perceptions are correlated to cognitive gains at least through the junior year in college ${ }^{40}$. However, not all studies support the chilly climate hypothesis, particularly those that do not explicitly question for discrimination, but instead compare male and female students for their responses to self-rating scales ${ }^{27,38}$.

The literature on discrimination within SME specifically is considerably more conclusive, however. Of those examining the "chilly climate" question ${ }^{5,10-13,27,33,37,41,42}$, only one did not find significant evidence of discrimination $\left(\operatorname{Strenta}^{27}\right)$. Like Drew \& Work ${ }^{38}$, Strenta does not explicitly question for discrimination.

There are two primary elements of the "chilly climate:" discrimination and the competitive/weedout culture. For the scope of this review, only the first will be examined in detail; indications of the latter are found in the quotes from Seymour ${ }^{8}$, above, and are elaborated upon in Seymour \& Hewitt ${ }^{13}$. This section investigates 2 questions:

1. Is discrimination more prevalent for women than for men?

2. What characterizes women's experiences of discrimination?

\section{The "Chilly Climate" — Differential Discrimination? There appears to be more} discrimination against women than men, at least for some institutions.

Four studies quantitatively compare men's and women's experiences with discrimination in engineering ${ }^{10,11,27,33}$. In Haines' examination ${ }^{10}$ of factors related to retention, women reported more sexual discrimination from faculty $(16 \%$ vs. $4 \%)$ than did their male peers. This differential discrimination (i.e., more towards females than males) had an effect size of .47-the largest of that study. Further, women also indicated more knowledge of others who experienced sexual discrimination from faculty $(17 \%$ vs. $25 \%)$, with an effect size of .20. The authors emphasize that 
the accumulation of micro-inequities points to the need for closer examination of those inequities - an ongoing focus for some of the "micro" analyses of this review ${ }^{12,37,41}$. Hughes $^{11}$ research on gender interaction in the classroom shows that, in comparison with male students, female students did report significantly less respect from the instructor and male students. Meinholdt ${ }^{33}$ employed 3 measures (sexism attitudes, awareness of sexual discrimination, and awareness of reverse discrimination) and found significant gender effects for the first $2(\mathrm{p}<.001$ for the first and $\mathrm{p}<.05$ for the second). I.e., using example items from the sexism attitudes scale, men are more likely than women to agree that "Men make better engineers than women" and to disagree that "It's all right for the woman to have a career and the man to stay home with the children" (p. 248).

Unlike the above 3 studies, Strenta ${ }^{27}$ investigated evidence of differential discrimination without explicitly referencing "discrimination" in the questionnaire. Instead, he surveyed students for their ratings of faculty responsiveness, accessibility, dedication to teaching, and motivation of students. Among engineers, only the last showed significance, and it was in favor of women. This study did allow students to comment at the end of the survey on any other factors related to their persistence. Of 533 comments, only 5 alleged overt or covert gender discrimination. Based primarily on this evidence, Strenta concludes that gender differences in self-confidence do "not seem to have to do with any discrimination, overt or covert, that might have been expressed in differential responses to the questions in this part of our survey" (p. 535).

How can we reconcile the above quantitative studies comparing men's and women's experiences with discrimination? There are a number of differences to consider. First is the question of "prompting"-it appears to be related to results. Prompting may result in a self-selection effect on questionnaire response rate which may bias results; Strenta thus omits it (as done also in Drew $\&$ Work $^{38}$ ). But, as outlined in Anderson ${ }^{5}$, Bergvall ${ }^{41}$, Seymour ${ }^{8}$, and Tonso ${ }^{37}$ many women do not have the conscious awareness to recognize discrimination even when they are the targets of it. Lacking awareness, discrimination is likely to go underreported, and even more unlikely to be reported if unprompted. As a possible explanation for this lack of awareness, Conefrey ${ }^{43}$ argues that women's prior socialization and lower self-esteem may discourage women from becoming aware of discrimination. Investigating discrimination without prompting also lacks facevalidity - an omission which requires considerable justification. Such justification may exist but it has not been outlined in detail. Reconciling these push and pull forces is an important area for future investigation.

A second difference is the institutional type: Strenta investigated highly selective institutions whereas Haines and Hughes investigated more mainstream institutions. It is certainly possible that discrimination may vary according to faculty and student selectivity.

While not a prime difference for the above three studies, the source of discrimination is a variable in many studies. Haines and Strenta prompt solely for student concerns about faculty, but not about fellow students. While differential discrimination from faculty has been found ${ }^{10,11}$, other studies have focused more on peer-related discrimination ${ }^{8,37,41}$.

Overall, given the findings of Haines ${ }^{10}$, Hughes ${ }^{11}$, and Meinholdt ${ }^{33}$ and the differences regarding 
Strenta $^{27}$, it appears there is evidence to support differential discrimination against women in engineering.

2. The "Chilly Climate" - Women's experiences: Women experience much subtle and some obvious discrimination in the form of discourse with male faculty and students. These women are not necessarily aware of being discriminated against.

The above three studies ${ }^{10,11,27}$ quantitatively compare men's and women's experiences. Other studies focus on the qualitative dimension of women's experiences.

Anderson's qualitative investigation ${ }^{5}$ of female engineering students revealed both subtle and obvious discrimination experiences for women, from both faculty and male peers. In their speech, some faculty assume the engineering field is exclusively male. For example, they may use men's names in hypothetical examples or use lewd humor directed towards college males that was uncomfortable for most college females: "I'm going to approach [this class] like I would a date and see how far I can get" (p. 111). Many women students experienced discrimination but apparently did not have the reflexive powers to recognize it: "...if a professor ever needs help with anything in the lab, he asks a male student. I suppose it's because it is physical, although it really doesn't require much strength" (p. 113). As in Seymour \& Hewitt, some male peers were jealous of female success: "The reason you got your job was because you were a girl, and they need girls!" (p. 114, italics in text). Such attitudes deprive women of equal status and tend to diminish their self-concept.

A group of studies examined gender interactions in SME and other group work ${ }^{12,37,42,44}$. Rosser ${ }^{42}$ reviewed the literature on women and minorities in group work. General group dynamics research has shown men tend to talk more than women, interrupt women much more frequently than viceversa, and that those interruptions are more frequently tangential or trivial and redirect the woman's statements. Women have a greater tendency to qualify their statements or use polite/deferential speech which may result in men more easily ignoring their statements.

Tonso $^{12,44}$ observed the discourse of engineering students involved in a sophomore small group design classes. Discriminatory discourse was observed against women, particularly when there was a single woman in the group. Semi-sexual double-entendre humor was practiced by men that appeared to make women uncomfortable (also seen in Anderson ${ }^{5}$ and Seymour ${ }^{8}$ ). Symbolic violence metaphors were used as normative by a majority of the men but not by women. Generally, roles and values of women were subordinate to men.

Tonso ${ }^{37}$ further examined gender interactions in $1^{\text {st }}$ year and senior design classes and in the larger campus community. Campus-wide, women were given cultural identities that were negative, invisible, and non-belonging. In group work, some women's contributions were undervalued and exploited by some men (also seen in Bergvall ${ }^{41}$ ). While most men did not hold sexist attitudes, a minority of powerful senior male students promoted hostility to women; other students and faculty were unable to check their power, resulting in a tolerance of sexism. Similar sexism not seen in $1^{\text {st }}$ year students, implying it was, to an extent, learned or dormant. Both male and female students showed limited awareness of sexual harassment and limited will to stop it. 
Seymour ${ }^{8}$ and Seymour \& Hewitt ${ }^{13}$ provide examples for women's discriminatory experiences in SME, many of which are given in the above section on self-efficacy. Recall the themes from some male students of male superiority and women's incompetence, and more subtle sexism experiences with male faculty. Seymour found that those women nonpersisters who experienced discrimination did not report that they left because of that ill-treatment. However, recall that many young women are unaware of discriminatory experiences even if directed against themselves. And, if discrimination instead acts more to wear down women's self-efficacy, and selfefficacy then in turn results in a loss of desire to persist, these women may not be aware of the complex effects of discrimination on their persistence. This domino effect is supported by Seymour's qualitative findings, and would be a prime area for quantitative/ structural investigation in future research.

Overall, women's experiences with discrimination originate from both faculty and students, are more often subtle than obvious, and occur primarily through discourse. But, women are often not consciously aware that they are experiencing discrimination.

\section{Integration: Gender Socialization, Ability, Self-Efficacy, And Discrimination}

What is the inter-relationship of the three primary factors of this review: ability, self-efficacy, and discrimination? How do we simultaneously explain the findings of this review:

1. Women have higher attrition than men in engineering.

2. Women have equal or better qualifications than men.

3. Women perform substantially the same as men.

4. Persistence for women and men is predicted by ability and self-efficacy, but is inconclusive with respect to gender.

5. In college, women have lower self-efficacy than their male peers.

6. Women have high self-efficacy entering college but lose a large portion of it soon after entry.

7. Women's self-efficacy is correlated to achievement and positive interactions with faculty.

8. Women experience discriminatory messages from male students and faculty which imply women's incompetence and non-belonging.

9. Women experience more discrimination than their male peers.

10. Women nonpersisters do not claim they left because of discrimination.

11. Women are not necessarily aware of their experience of discrimination.

There are likely many complex mechanisms governing the above findings. The following proposed theoretical history may provide one coherent explanation for the above findings. Most of the elements of this history are found in the literature of this review, particularly, Seymour ${ }^{8}$, Leslie $^{17}$, Schaefers ${ }^{4}$, Felder ${ }^{18}$, and Hackett ${ }^{19}$; this review integrates and condenses these elements.

- Socialization processes in junior high and high school imprint lower intrinsic SME selfefficacy upon women than men.

- Generous high school teacher support offsets this lower intrinsic self-efficacy and allows women to develop a contingently high SME self-efficacy, with equal qualifications and abilities to men (Findings 2 and 3). But, their SME self-efficacy is dependent upon teacher's support. 
- Entering college, this support is not only withdrawn, faculty raise expectations and expect students to prove themselves without support. In response to this disturbance, without awareness that it is "not personal," and with lower intrinsic SME self-efficacy, women's expressed self-efficacy drops rapidly (Finding 6). Men respond with less maladjustment because they understand competition and have more intrinsic self-efficacy (Finding 5).

- Unless women regain self-efficacy via faculty interactions and/or their own high performance (Finding 7), their low self-efficacy results in higher risk of non-persistence (Finding 1 and 4). Those (fewer) men with equally low self-efficacy are at equal risk of non-persistence (Finding 4). That is, self-efficacy may be a mediator between gender and persistence (Findings 1, 2, 3, 4, and 5).

- Compounding these effects, subtle and obvious differential discrimination messages from some male faculty and students further call into question women's place and ability in engineering (Findings 8 and 9), which further reduces their self-efficacy.

- Lowered self-efficacy due to discrimination differentially influences women to not persist. Self-efficacy may also be a mediator between discrimination and persistence (Findings 1, $4,5,8$, and 9).

- As 1) discrimination does not directly affect persistence, but indirectly via self-efficacy, and 2) women are not necessarily aware of the discrimination they experience, women do not claim to leave engineering because of discrimination (Findings 10 and 11).

This proposed theoretical model is based on the research literature and does integrate the primary findings, but it is tentative. It is not intended to explain all potential forces on women's persistence in engineering. Further research is necessary to untangle the complex mechanisms, empirically testing the claims made.

\section{Conclusions}

The persistence rate of women in SME is considerably lower than that of men, despite equal or better academic credentials. Studies have shown the persistence rates of women and men in SME majors are well predicted by performance and self-efficacy, regardless of gender, and that women tend to lose confidence in their scientific abilities from the start of their $1^{\text {st }}$ year, despite adequate or better performance levels. Discrimination is more prevalent for women than men and sends messages that women are not competent and do not belong in engineering. These factors coalesce to lower the self-efficacy of women, which then places them at increased risk of nonpersistence. A theoretical model is offered that ascribes two mediational roles for selfefficacy. These structural models may be examined in future research. Interventions to improve the retention of women in engineering may be more successful if the structural characteristics of the forces on women are better understood. Should the proposed model prove valid, it would provide insight towards more effective interventions.

Whether this model is valid or not, interventions will likely require more than surface-level improvements in engineering education. According to Leslie ${ }^{17}$ structural changes in higher education are necessary to encourage rather than discourage students to pursue science and engineering. Leslie holds that most SME faculty are simply unaware of the detrimental effects of their programs. Seymour ${ }^{8}$ similarly recommends two simultaneous interventions: first, to educate girls to rely less on external sources of motivation and reassurance so that they may survive in the 
current SME system, and second, to change the SME system so that it is more accommodating to non-masculine personalities.

For economic, productivity, social, and ethical motivations, increasing the representation of women in engineering is an important priority. Efforts to increase their representation have not met with large success. Educators, as core formation agents of engineering culture, need to understand the complex structures and dynamics of women's experiences in engineering if more effective changes are to be found. From this understanding, with effort and courage, systemic reform may allow greater equity in engineering education.

\section{Bibliography}

1. National Science Foundation, Women, minorities, and persons with disabilities. Vol. 00-327. 2000, Arlington: NSF.

2. Farrell, E.F., "Engineering a warmer welcome for engineering students," in The Chronicle of Higher Education, 2002, p. A31-A32.

3. Heller, R.S. and C.D. Martin, "Attracting young minority women to engineering and science: Necessary characteristics for exemplary programs," IEEE Transactions on Education, 1994, 37: 8-13.

4. Schaefers, K.G., D.L. Epperson, and M.M. Nauta, "Women's career development: Can theoretically derived variables perdict persistence in engineering majors?," Journal of Counseling Psychology, 1997, 44: 173-183.

5. $\quad$ Anderson, V., "How engineering education shortchanges women," Journal of Women and Minorities in Science and Engineering, 1994, 1: 99-121.

6. Bergvall, V., S. Sorby, and J. Worthen, "Thawing the freezing climate for women in engineering education: Views from both sides of the desk," Journal of Women and Minorities in Science and Engineering, 1994, 1: 323-346.

7. National Research Council, Transforming undergraduate education in science, mathematics, engineering, and technology. 1999, Washington, DC: National Academy Press.

8. Seymour, E., "The loss of women from science, mathematics and engineering undergraduate majors: An explanatory account," Science Education, 1995, 79: 437-473.

9. Dececchi, T., M.E. Timperon, and B.B. Dececchi, "An analysis of male/female academic performance for engineering students at The Royal Military College of Canada," Journal of Engineering Education, 1996, 85: 133-141.

10. Haines, V.A., J.E. Wallace, and M.E. Connon, "Exploring the gender gap in engineering: A respecification and test of the hypothesis of cumulative advantages and disadvantages," Journal of Engineering Education, 2001, 90: 677-684.

11. Hughes, W.J., "Perceived gender interaction and course confidence among undergraduate science, mathematics, and technology majors," Journal of Women and Minorities in Science and Engineering, 2000, 6: 155-167.

12. Tonso, K.L., "The impact of cultural norms on women," Journal of Engineering Education, 1996, 85: 217 225.

13. Seymour, E. and N.M. Hewitt, Talking about leaving: Why undergraduates leave the sciences. 1997, Boulder, CO: Westview Press.

14. Adelman, C. and U.S. Dept. of Education, Women and men of the engineering path: a model for analyses of undergraduate careers. 1998, Washington, DC: U.S. Dept. of Education: National Institute for Science Education.

15. Astin, A.W., What matters in college: Four critical years revisited. 1993, San Francisco: Jossey-Bass.

16. Humphreys, S.M. and R. Freeland, "Retention in engin eering: A study of freshman cohorts," 1992, cited 
in Adelman, 1998. University of California: Berkeley, CA.

17. Leslie, L.L., G.T. McClure, and R.L. Oaxaca, "Women and minortities in science and engineering: A life sequence analysis," The Journal of Higher Education, 1998, 69: 239-276.

18. Felder, R.M., G.N. Felder, M. Mauney, C.E. Hamrin, and E.J. Dietz, "A longitudinal study of engineering student performance and retention. III. Gender differences in student performance and attitudes," Journal of Engineering Education, 1995, 84: 151-163.

19. Hackett, G., N.E. Betz, J.M. Casas, and I.A. Rocha-Singh, "Gender, ethni city, and social cognitive factors predicting the academic achievement of students in engineering," Journal of Counseling Psychology, 1992, 39: 527-538.

20. McClelland, L., "Students entering science, mathematics, and engineering majors as fall freshmen, 19801988," 1993, Unpublished data provided by L. McClelland, (cited in Seymour \& Hewitt, 1997). University of Colorado, Office of Research and Information: Boulder.

21. Hyde, J.S., "Meta-analysis and the psychology of gender differences," Signs: Journal of Women in Culture and Society, 1990, 16: 55-73.

22. Devon, R., R. Engel, and G. Turner, "The effects of spatial visualization skill training on gender and retention in engineering," Journal of Women and Minorities in Science and Engineering, 1998, 4: 371380 .

23. Sorby, S.A., C. Leopold, and R. Gorska, "Cross-cultural comparisons of gender differences in the spatial skills of engineering students," Journal of Women and Minorities in Science and Engineering, 1999, 5: 279-91.

24. Grandy, J., "Gender and ethnic differences in the experiences, achievements, and expectations of science and engineering majors," Journal of Women and Minorities in Science and Engineering, 1997, 3: 119143.

25. Takahira, S., D.J. Goodings, and J.P. Byrnes, "Retention and performance of male and female engineering students: an examination of academic and environmental variables," Journal of Engineering Education, 1998, 87: 297-304.

26. Sax, L.J., "Retaining tomorrow's scientists: Exploring the factors that keep male and female college students interested in science careers," Journal of Women and Minorities in Science and Engineering, 1994, 1: 45-61.

27. Strenta, A.C., R. Elliott, R.M. Adair, M., and J. Scott, "Choosing and leaving science in highly selective institutions," Research in Higher Education, 1994, 35: 513-47.

28. Brainard, S.G. and L. Carlin, "A six year longitudinal study of undergraduate women in engineering and science," Journal of Engineering Education, 1998, 87: 369-376.

29. Bandura, A., "Self-efficacy: Toward a unifying theory of behaviorial change," Psychological Review, 1977, 84: 191-214.

30. Lent, R.W., S.D. Brown, and K.C. Larkin, "Comparison of three theoretically derived variables in predicting career and academic behavior: Self-efficacy, interest congruence, and consequency thinking," Journal of Counseling Psychology, 1987, cited in Hackett, 1992, 34: 293-298.

31. Hackett, G. and N.E. Betz, "A self-efficacy approach to the career development of women," Journal of Vocational Behavior, 1981, 18: 326-339.

32. Besterfield-Sacre, M.B., M. Moreno, L.J. Shuman, and C.J. Atman, "Gender and ethnicity differences in freshman engineering student attitudes: A cross-institutional study," Journal of Engineering Education, 2001, 90: 477-490.

33. Meinholdt, C. and S.L. Murray, "Why aren't there more women engineers?," Journal of Women and Minorities in Science and Engineering, 1999, 5: 239-63.

34. Besterfield-Sacre, M.B., C.J. Atman, and L.J. Shuman, "Characteristics of freshman engineering students: Models for determining student attrition in engineering," Journal of Engineering Education, 1997, 86: 139-149.

35. Arnold, K.D., "Values and vocations: The career aspirations of academically-gifted females in the first five years after high schol," in Annual Meeting of the American Educational Research Association, 1987, cited in Seymour, 1995, Washington, DC.

36. Nauta, M.M., D.L. Epperson, and J.H. Kahn, "A multiple-groups analysis of predictors of higher level career aspirations among women in math, science, and engineering majors," Journal of Counseling Psychology, 1998, 45: 483-496.

Proceedings of the 2003 American Society for Engineering Education Annual Conference \& Exposition Copyright $(\mathrm{C}$ 2003, American Society for Engineering Education 
37. Tonso, K.L., "Engineering gender-gendered engineering," Journal of Women and Minorities in Science and Engineering, 1999, 5: 365-405.

38. Drew, T. and G. Work, "Gender based difference in perceptions of experiences in higher education," The Journal of Higher Education, 1998, 69: 542-555.

39. Crawford, M. and M. MacLeod, "Gender in the college classroom: An assessment of the "chilly climate" for women," Sex Roles, 1990, 23: 101-122.

40. Whitt, E.J., M.I. Edison, E.T. Pascarella, A. Nora, and P.T. Terenzini, "Women's perceptions of a "chilly climate" and cognitive outcomes in college: Additional evidence," Journal of College Student Development, 1999, 40: 163-177.

41. Bergvall, V., "Constructing and enacting gender through discourse: Negotiating multiple roles as female engineering students," in Rethinking language and gender research: Theory and practice, V. Bergvall, Editor, 1996, Longman: NY. p. 173-201.

42. Rosser, S.V., "Group work in science, engineering, and mathematics: Consequences of ingnoring gender and race," College Teaching, 1998, 46: 82-88.

43. Conefrey, T., "Sexual discrimination and women's retention rates in science and engineering programs," Feminist Teacher, 2001, 13: 170-192.

44. Tonso, K.L., "Student learning and gender," Journal of Engineering Education, 1996, 85: 143-150.

\section{Biographical Information}

DAVID MALICKY has a Ph.D. from the Univ. of Michigan in Mechanical Engineering and is currently completing a Masters/Post-Doc in Counseling Psychology at the Univ. of Kansas. He was formally an assistant professor of Mech. Engr. at Valparaiso Univ. His interests include the development of engineering students as balanced individuals and the development and retention of women and minorities in engineering. Email: dmalicky@ku.edu 